\title{
In Vitro Screening of Different Potato Genotypes for Salinity Tolerance
}

\author{
El-Magawry, N.A. ${ }^{1}$; F.H. Mohamed ${ }^{2}$; M.W.M. Elwan ${ }^{2 *}$; K.E. Abdel-Hamid ${ }^{2}$ and M.M. Abdel-Salam ${ }^{1}$ \\ ${ }^{1}$ Agric. Research Center, Giza, Egypt \\ ${ }^{2}$ Department of Horticulture, Faculty of Agriculture, Suez Canal University, Ismailia, Egypt
}

Received: $22 / 4 / 2015$

\begin{abstract}
In vitro screening of several potato genotypes (29 cultivars and lines) to salinity stress was conducted. Single node explants were evaluated on the basis of their growth, microtuberization and biochemical analysis under different concentrations of salt $(\mathrm{NaCl})$ stress. In most cases, increasing $\mathrm{NaCl}$ in the tissue culture medium resulted in reduction in plantlet growth, rooting and microtuberization potential in varying degrees, depending on the cultivar. Some genotypes such as Oceania, 97f-267 and Picasso produced higher number of microtuber under $150 \mathrm{mM} \mathrm{NaCl}$ than control. Generally, 97f-267, Oceania and Universa genotypes were ranked among salt tolerant, while Nicola, Safran, Diamant were ranked as moderate and Elodi, Triomph, Marabel, Bolista and 99-981 were ranked as salt sensitive based on morphological characters. Photosynthetic pigments decreased under salt stress, however, Universa and Safran maintained higher chlorophyll content under salt conditions $(100 \mathrm{mM} \mathrm{NaCl})$ comparing with control $(0.0 \mathrm{mM} \mathrm{NaCl})$ plantlets. Also, Universa and Safran accumulated more proline and free amino acids under stress treatment comparing with control, than other tested genotypes. The activities of antioxidant enzymes were different among potato genotypes. In this respect, Universa and Safran had higher catalase (CAT) activity under salt stress (100 mM NaCl) than control treatment $(0.0 \mathrm{mM} \mathrm{NaCl})$, however, superoxide dismutase (SOD) activity was not good indicator for salinity tolerance in the tested potato genotypes.
\end{abstract}

Keywords: Solanum tuberosum L., tissue culture, abiotic stress, $\mathrm{NaCl}$, microtuberization.

\section{INTRODUCTION}

Potato (Solanum tuberosum L.) is one of the most important vegetable crops in Egypt for local consumption and exportation. The total area devoted for production in the year 2013 in Egypt was 300,662 fad., with total production of about 4,500,000 tons (average 14,96 ton/fad.). Worldwide, potato is the fourth most important crop, with an annual production of 325 million tons, (FAO state, 2012). In Egypt, about $72,918.75$ tons of tuber seeds were imported in the year 2012 for summer plantation from European countries. The tuber seeds costs were about 6.8 million Dollars.

Salinity is a serious problem for commercial agriculture worldwide where about one billion ha are affected by salinity (Christiansen, 1982). In this regards, potato is classified as moderately salt sensitive crop (Katerji et al., 2002). However, more information are needed regarding the tolerant or sensitive genotypes to salt stress, due to the significant variation in salt tolerance among potato genotypes (Kharis et al., 1998). Abiotic stresses, such as high salinity often result in significant losses to the yields of economically important crops such as potato (Ahmed and Rashid, 1990).

Examining the field performance of potato genotypes under a salinity stress is the usual method for evaluation, but, the results are often inconclusive. Field trial is normally associated with the spatial distribution of salt, non-uniform moisture availability and temperature fluctuations during the growing season. This method involves considerable space, time, labor, equipment and planting material resources (Arvin and Donnelly, 2008). Therefore, in vitro screening of the new currently grown genotypes represents valuable tool as alternative to field trials. Furthermore, a highly significant correlation was found between in vitro growth parameters and field performance of ten potato clones studied by Morpurgo (1991). In vitro screening for salinity tolerance was published (Polturi and Prasad, 1993; Zhang and Donnely, 1997; Khenifi et al., 2011) with limited number of potato genotypes based on plant growth or microtuber formation. Few literatures examined large number of genotypes (Khrais et al., 1998) based on plantlet growth.

Plants constantly exposed to capricious conditions have adapted at the molecular, cellular, physiological and biochemical levels, enabling them to survive and cope with adverse environmental stresses. Bouaziz et al. (2012) and Marcek et al. (2014) reported an increase in proline accumulation. Also, Asensia-Fabado et al. (2014) found increase in total free amino acids with salinity stress. However, Potluri and Prasad (2004) reported that the proline accumulation under salinity stress was cultivar-dependent. In most cases, antioxidant enzymes activity such as superoxide dismutase (SOD) and catalase (CAT) increased in salt tolerant potato genotypes (Daneshmand et al., 2010; Sajid and Faheem, 2014), however, in another study, the activity of SOD decreased with salinity stress (Zhang et al., 2007).

In Egypt, several new potato cvs were introduced to farmers during the past decades, of these; more than 30 cultivars are micro-propagated in the Plant Tissue Culture Lab, of the Horticulture Department of Suez Canal University and represent a valuable germplasm stock material to be evaluated against several a biotic stresses such as salinity stress.

The objective of the present study was directed towards screening large number of newly introduced potato genotypes and breeding lines, as well as some potato genotypes already cultivated in Egypt, for salinity stress in vitro using $\mathrm{NaCl}$ as stress agent. The sub aim of the study was examination of the physiological and biochemical changes associated with the tolerance or sensitivity to salinity stress. 


\section{MATERIALS AND METHODS}

The current investigation was conducted at the Plant Tissue Culture laboratory of the Horticulture Department, Suez Canal University, Ismailia, Egypt during the period of 2012 to 2014. This experiment was conducted to study the effect of salinity stress on morphological (shoot and root growth characters) and microtuber-forming capacity of different potato cultivars under in vitro conditions.

\section{Plant materials and culture conditions}

The experiment included 29 potato genotypes of three maturity groups, early, moderate, and late maturity genotypes. Six early genotypes (Safran, Margod, Universa, Alaska, Spunta and Elodi), seven mid-early genotypes (Triomph, Lady Rosetta, Nicola, Fridor, Naga, Oceania and Diamant), late maturity (Agria) and seven potato lines $(97-980,94 \mathrm{f}, 97 \mathrm{~F}-267,94 \mathrm{f}-8101,96 \mathrm{f}-$ 25-25, 95k-94 and 99-981), German varieties (Jelly, Presto and Marabel) and locally-grown old cultivars (Picasso, Proventa, Arinda, Bolista and Sante).

The locally-grown cultivars were kindly provided by The Vegetable Research Department, Horticulture Research Institute, Giza, Egypt. The newly introduced genotypes were kindly provided by the seed potato production support project, Ministry of Agriculture, central administration for seed testing and certification funded by the French Food Aid Counterpart fund.

Potato tubers from the different genotypes were cultivated in pots containing wet vermiculite under glasshouse conditions until sprouting. Sprout of $5 \mathrm{~cm}$ long were collected for sterilization with $10 \%$ commercial bleach (2.5\% hypochlorite) for $10 \mathrm{~min}$, and then washed with sterile distilled water three times in a laminar air flow hood. Meristem tip explants $(0.3 \mathrm{~mm})$ were excised from sprout shoot tips under binuclear microscope. Cultures were incubated at $25 \pm 2^{\circ} \mathrm{C}$ with $16 / 8 \mathrm{~h}$ day/night at $40 \mu \mathrm{mal} \mathrm{m}^{2} \mathrm{~s}^{1}$ photon flux density (cool white fluorescent light). For micropropagation, MS (Murashige and Skoog, 1962) basal salts and vitamin (Duchefa Biochemi, the Netherlands) was used, supplemented with $3 \%$ sucrose and $0.7 \%$ agar. The medium was adjusted to $5.8 \mathrm{pH}$ before the addition of agar.

In vitro grown plants were propagated by subculture with 4 weeks interval for three sub-cultures before starting the experiment. Ten single node explants (about $1 \mathrm{~cm}$ ) were sub-cultured into $350 \mathrm{ml}$ ca. jars containing $30 \mathrm{ml}$ MS free-medium. The proliferated cultures of 4 weeks old and approximately $10 \mathrm{~cm}$ long, avoiding the top and bottom node segments were used as the starting materials for subsequent trials. Media were sterilized by autoclaving at $121^{\circ} \mathrm{C}$ and $1.05 \mathrm{~kg} / \mathrm{cm}^{2}$ for $20 \mathrm{~min}$., then dispensed into the tissue culture jars.

After three subcultures, ten single-node explants from the tested potato genotypes were transferred to MS medium containing $0.0,50,100$ and $150 \mathrm{mM} \mathrm{NaCl}$ with five replicates for each treatment. After 6 weeks, plantlet samples were taken from each salt treatment for morphological data, such as plantlet length and root number. The percentage of growth under $150 \mathrm{mM} \mathrm{NaCl}$ as relative to the control $(0.0 \mathrm{mM} \mathrm{NaCl})$ was estimated for each growth character.
For microtuberization, $30 \mathrm{ml}$ sterilized liquid MS medium amended with high sucrose level (80 g/l) were added to each jar containing the growing plantlets. Cultures were incubated in the dark at $18-20^{\circ} \mathrm{C}$ for 2 months. Microtubers produced from each treatment were harvested and data were taken on number and weight (yield) of microtuber/jar and the average single microtuber weight were calculated by dividing weight/number. Percent of tuber formation under the highest $\mathrm{NaCl}$ level $(150 \mathrm{mM})$ was also calculated as relative to the control.

\section{Biochemical analysis of potato plant grown under in} vitro salinity stress conditions.

Based on the morphological characters only seven potato genotypes were chosen for biochemical analysis as representing, 1) Salt tolerant genotypes (Universa and Agria). 2) Moderately tolerant genotypes (cvs. Nicola, Sufran and Triomph), and 3) salt sensitive genotypes (Bolista and Diamant). The biochemical analysis were made only in plantlets exposed to the salt at level 100 $\mathrm{mM}$ in comparison with control plantlets.

1-Chlorophyll (Chl) and carotenoid contents were determined according to the method of Lichtenthaler (1987).

2- Free total amino acids were colorimetrically assayed by ninhydrin reagent at $570 \mathrm{~nm}$ according to the method described by Lee and Takabashi (1966).

3-Proline was estimated using the method described by Sadasivam and Manickam (1991).

4- Enzymes: The plantlet samples (weight 0.1-0.4g) were prepared for enzyme activity as described by $\mathrm{Ni}$ et al. (2001).

4-a. Superoxide dismutase (SOD) activity was determined spectrophotometrically at $560 \mathrm{~nm}$ (U\V Spectrophotometer spectronic 1201, Milton Roy, U.S.A) as described by the method of Nishikimi et al. (1972).

4-b. Catalase (CAT) activity was measured spectrophotometrically at $510 \mathrm{~nm}$ as described by the method of Aebi (1984).

\section{Statistical analysis}

The experiment was conducted twice with five replicates each, using a randomized complete block design in factorial fashion. Data were combined and subjected to ANOVA using CoStat program and the means were separated by Duncan's multiple-range test at $5 \%$ level.

\section{RESULTS}

\section{Effect of salinity on plantlet growth:}

Results revealed that increasing $\mathrm{NaCl}$ in the medium resulted in significant decrease in plantlet length (Table 1) as an average over all 29 genotypes tested. Average plantlet length was the highest under control $(0.0 \mathrm{NaCl})$ and $50 \mathrm{mM} \mathrm{NaCl}(5.97$ and $5.86 \mathrm{~cm}$, respectively). However, shoot length declined significantly at $100 \mathrm{mM}$ (average $3.0 \mathrm{~cm}$ ) and showed the highest reduction at $150 \mathrm{mM} \mathrm{NaCl}$ (average $1.38 \mathrm{~cm}$ ) which represented $77 \%$ reduction compared to the control.

Rooting was also affected with salinity increases in the tissue culture medium. High salt level, dramatically 
affected rooting of potato plantlet in vitro (Table 2). As an average over all tested genotypes, the highest root number was detected under $\mathrm{NaCl}$-free medium (average about 2.0 roots/plantlet). Root number decreased significantly with the increase in $\mathrm{NaCl}$ level, recording an average 1.76 roots at $50 \mathrm{mM} \mathrm{NaCl}, 1.1$ roots at 100 $\mathrm{mM}$, and only average 0.28 root/plantlet at $150 \mathrm{mM}$ $\mathrm{NaCl}$ (about $86 \%$ decline over the control).

Potato genotypes showed significant differences in their shoot growth characters, as tested over the $\mathrm{NaCl}$ treatments. With respect to plantlet length, the differences among potato genotypes were found significant (Table 1). Under the conditions of this experiment, the ANOVA results indicated that $\mathrm{cv}$. Arinda, followed by Lady Rosetta, line 97f. 25.25, 95$94 \mathrm{k}$ and cv. Agria recorded the highest shoot length as an average over all the tested $\mathrm{NaCl}$ levels, while the line 99-981 and cv. Jelly recorded the least shoot length. Genotypes were significantly different in root number/plantlet (Table 2). The highest recorded root number was found in cv. Safran and Picasso, followed by Bolista (average 2.7, 2.5 and 2.18 roots, respectively), while the lowest roots were found in the two genotypes, $94 \mathrm{f}-8101$ and Sante.

Table (1). Effect of $\mathrm{NaCl}$ concentration on the in vitro shoot (plantlet) length in 29 potato genotypes.

\begin{tabular}{|c|c|c|c|c|c|c|c|c|c|c|c|}
\hline \multirow{2}{*}{$\begin{array}{c}\text { CV } \\
\text { Safran }\end{array}$} & \multicolumn{2}{|c|}{0.0} & \multicolumn{2}{|c|}{50} & \multicolumn{2}{|c|}{100} & \multicolumn{2}{|c|}{150} & \multirow{2}{*}{\multicolumn{2}{|c|}{ Mean cv. }} & \multirow{2}{*}{$\begin{array}{c}\begin{array}{c}\text { \% of } \\
\text { control* }\end{array} \\
21.151\end{array}$} \\
\hline & & & \multicolumn{6}{|c|}{ Shoot length (cm) } & & & \\
\hline & 6.318 & $\mathrm{f}-\mathrm{o}^{* *}$ & 6.227 & h-p & 2.318 & H-Q & 1.336 & $\mathrm{~N}-\mathrm{V}$ & 4.050 & $e-h$ & \\
\hline $94 f-8101$ & 5.391 & $\mathrm{~m}-\mathrm{v}$ & 5.773 & $\mathrm{j}-\mathrm{s}$ & 1.773 & $\mathrm{~K}-\mathrm{V}$ & 0.727 & $\mathrm{~S}-\mathrm{V}$ & 3.416 & h-m & 13.491 \\
\hline Margod & 5.955 & i-r & 6.955 & $\mathrm{~d}-1$ & 1.682 & $\mathrm{~L}-\mathrm{V}$ & 0.591 & $\mathrm{~T}-\mathrm{V}$ & 3.795 & $f-j$ & 9.924 \\
\hline Universa & 6.091 & $h-q$ & 4.545 & $\mathrm{r}-\mathrm{B}$ & 2.682 & $\mathrm{~F}-\mathrm{N}$ & 2.273 & H-R & 3.898 & $e-i$ & 37.313 \\
\hline Alaska & 5.364 & $\mathrm{~m}-\mathrm{v}$ & 4.136 & $v-E$ & 2.273 & H-R & 0.864 & $\mathrm{R}-\mathrm{V}$ & 3.159 & $\mathrm{j}-\mathrm{n}$ & 16.102 \\
\hline Spunta & 7.273 & $c-i$ & 6.455 & $\mathrm{f}-\mathrm{n}$ & 3.318 & A-J & 1.182 & $\mathrm{O}-\mathrm{V}$ & 4.557 & de & 16.250 \\
\hline Elodi & 7.464 & $b-h$ & 5.591 & $1-\mathrm{u}$ & 0.636 & $\mathrm{~S}-\mathrm{V}$ & 0.545 & UV & 3.559 & h-1 & 7.308 \\
\hline $97 f-25-25$ & 5.773 & $\mathrm{j}-\mathrm{s}$ & 12.000 & $\mathrm{a}$ & 3.591 & $\mathrm{y}-\mathrm{H}$ & 2.018 & $\mathrm{I}-\mathrm{T}$ & 5.845 & $a b$ & 34.961 \\
\hline Triomph & 5.682 & $1-\mathrm{s}$ & 4.818 & $\mathrm{p}-\mathrm{z}$ & 0.591 & $\mathrm{~T}-\mathrm{V}$ & 0.727 & $\mathrm{~S}-\mathrm{V}$ & 2.955 & $\mathrm{k}-\mathrm{n}$ & 12.800 \\
\hline $\begin{array}{l}\text { Lady } \\
\text { Rosetta }\end{array}$ & 7.136 & $\mathrm{c}-\mathrm{k}$ & 8.182 & b-e & 7.682 & b-g & 0.636 & $\mathrm{~S}-\mathrm{V}$ & 5.909 & $\mathrm{ab}$ & 8.917 \\
\hline 95k-94 & 7.500 & b-h & 8.909 & $\mathrm{~b}$ & 4.227 & $\mathrm{t}-\mathrm{D}$ & 2.045 & $\mathrm{I}-\mathrm{S}$ & 5.670 & $a b$ & 27.273 \\
\hline Oceania & 3.045 & D-L & 5.682 & $1-\mathrm{s}$ & 7.318 & $c-i$ & 1.136 & $\mathrm{O}-\mathrm{V}$ & 4.295 & efg & 37.313 \\
\hline Nicola & 4.545 & $r-B$ & 4.182 & $\mathrm{u}-\mathrm{E}$ & 1.773 & $\mathrm{~K}-\mathrm{V}$ & 0.682 & S-V & 2.795 & mno & 15.000 \\
\hline Fridor & 5.636 & $1-\mathrm{t}$ & 5.273 & m-w & 4.136 & $v-E$ & 1.091 & $\mathrm{P}-\mathrm{V}$ & 4.034 & $e-h$ & 19.355 \\
\hline Naga & 5.955 & i-r & 5.045 & $n-x$ & 2.955 & D-M & 0.682 & S-V & 3.659 & $f-k$ & 11.450 \\
\hline Diamant & 4.745 & $\mathrm{q}-\mathrm{A}$ & 6.455 & $\mathrm{f}-\mathrm{n}$ & 1.909 & J-U & 0.500 & UV & 3.402 & $h-m$ & 10.536 \\
\hline Agria & 7.727 & $b-f$ & 5.364 & $m-v$ & 4.955 & o-y & 4.000 & $\mathrm{v}-\mathrm{F}$ & 5.511 & $a b$ & 51.765 \\
\hline $99-981$ & 3.455 & z-I & 3.545 & $y-H$ & 1.091 & P-V & 0.727 & S-V & 2.205 & o & 21.053 \\
\hline $97-980$ & 3.864 & w-G & 4.727 & $\mathrm{q}-\mathrm{A}$ & 1.000 & $\mathrm{P}-\mathrm{V}$ & 1.318 & $\mathrm{~N}-\mathrm{V}$ & 2.727 & mno & 34.118 \\
\hline $94 f$ & 4.955 & o-y & 4.500 & $\mathrm{~s}-\mathrm{C}$ & 1.682 & L-V & 0.364 & $\mathrm{~V}$ & 2.875 & $1-0$ & 7.339 \\
\hline Jelly & 4.691 & $\mathrm{q}-\mathrm{A}$ & 3.182 & B-K & 1.455 & $\mathrm{~N}-\mathrm{V}$ & 0.591 & $\mathrm{~T}-\mathrm{V}$ & 2.480 & no & 12.597 \\
\hline Presto & 5.727 & $\mathrm{k}-\mathrm{s}$ & 6.273 & g-o & 3.091 & C-L & 2.364 & H-P & 4.364 & ef & 41.270 \\
\hline Marabel & 7.182 & $c-j$ & 8.545 & bc & 3.700 & $\mathrm{x}-\mathrm{H}$ & 1.727 & $\mathrm{~L}-\mathrm{V}$ & 5.289 & $\mathrm{bc}$ & 24.051 \\
\hline $97 f-267$ & 6.591 & $f-m$ & 5.364 & $m-v$ & 6.318 & f-o & 2.764 & E-N & 5.259 & bcd & 41.931 \\
\hline Picasso & 6.318 & f-o & 6.864 & e-1 & 4.182 & $\mathrm{u}-\mathrm{E}$ & 0.909 & Q-V & 4.568 & cde & 14.388 \\
\hline Provinta & 8.364 & bcd & 6.636 & $\mathrm{f}-\mathrm{m}$ & 1.727 & L-V & 1.591 & $\mathrm{M}-\mathrm{V}$ & 4.580 & cde & 19.022 \\
\hline Arinda & 8.909 & $\mathrm{~b}$ & 6.955 & $\mathrm{~d}-1$ & 4.818 & $\mathrm{p}-\mathrm{z}$ & 4.000 & $v-F$ & 6.170 & $\mathrm{a}$ & 44.898 \\
\hline Bolista & 6.318 & $f-o$ & 3.955 & $\mathrm{v}-\mathrm{G}$ & 2.545 & $\mathrm{G}-\mathrm{O}$ & 1.545 & $\mathrm{M}-\mathrm{V}$ & 3.591 & g-1 & 24.460 \\
\hline Sante & 5.318 & $\mathrm{~m}-\mathrm{v}$ & 3.818 & $\mathrm{x}-\mathrm{G}$ & 2.364 & H-P & 1.227 & $\mathrm{O}-\mathrm{V}$ & 3.182 & i-n & 23.077 \\
\hline Mean $\mathrm{NaCl}$ & 5.976 & $\mathbf{a}$ & 5.861 & $\mathbf{a}$ & 3.03 & b & 1.385 & c & & & \\
\hline
\end{tabular}


Growth of potato plantlets was significantly affected by the interaction of $\mathrm{NaCl} \times$ genotypes. With regard to plant length, results of the interaction revealed that the highest shoot length was recorded on genotype 97f.25.25 $(12.0 \mathrm{~cm})$ at $50 \mathrm{mM} \mathrm{NaCl}$, followed by cv. Arinda at the control treatment $(0.0 \mathrm{NaCl})$ with average $8.9 \mathrm{~cm}$. At $100 \mathrm{mM} \mathrm{NaCl}$, the cv. Lady Rosetta had the highest plantlet height (ave. $7.68 \mathrm{~cm}$ ), while the cv. Arinda recorded the highest plantlet height $(4.0 \mathrm{~cm})$ over all other genotypes at $150 \mathrm{mM} \mathrm{NaCl}$ (Table 1).
Regarding of root number per plantlet, the highest recorded number was found with $\mathrm{cv}$. Bolista at 0.0 $\mathrm{NaCl}$, followed by cvs Naga, Picasso, Elodi, Arinda and 97F.25-25. The cv Oceania formed more roots at 100 $\mathrm{mMNaCl}$ than at 0.0 or $50 \mathrm{mM}$, but failed to form roots at $150 \mathrm{mM} \mathrm{NaCl}$, however Safran formed a comparable root number at $150 \mathrm{mM} \mathrm{NaCl}$ as the control (Table 2).

These results indicated differences in potato genotype responses to the $\mathrm{NaCl}$ salinity stress tested under the condition of this experiment.

Table (2). Effect of $\mathrm{NaCl}$ concentration on root number per plantlet in 29 potato genotypes

\begin{tabular}{|c|c|c|c|c|c|c|c|c|c|c|c|}
\hline \multirow{3}{*}{$\begin{array}{l}\text { CV } \\
\text { Safran }\end{array}$} & \multicolumn{2}{|c|}{0.0} & \multicolumn{2}{|c|}{50} & \multicolumn{2}{|c|}{100} & \multicolumn{2}{|c|}{150} & \multirow{2}{*}{\multicolumn{2}{|c|}{ Mean cv. }} & \multirow{3}{*}{$\begin{array}{c}\begin{array}{c}\text { \% of } \\
\text { control* }\end{array} \\
107.14\end{array}$} \\
\hline & \multicolumn{8}{|c|}{ Ave. Root no. per plantlet } & & & \\
\hline & 2.545 & $\mathrm{~g}-\mathrm{m} * *$ & 3.273 & b-e & 2.545 & g-m & 2.727 & e-k & 2.773 & $\mathrm{a}$ & \\
\hline $94 f-8101$ & 0.545 & $y-D$ & 0.182 & $\mathrm{D}$ & 0.273 & $\mathrm{BCD}$ & 0.000 & $\mathrm{D}$ & 0.250 & $\mathrm{n}$ & 0.00 \\
\hline Margod & 1.455 & $r-v$ & 1.100 & $\mathrm{u}-\mathrm{y}$ & 0.000 & $\mathrm{D}$ & 0.000 & $\mathrm{D}$ & 0.380 & $\mathrm{~m}$ & 0.00 \\
\hline Universa & 2.182 & $k-p$ & 0.364 & A-D & 1.455 & $r-v$ & 0.000 & $\mathrm{D}$ & 1.000 & hij & 0.00 \\
\hline Alaska & 1.182 & $t-x$ & 0.727 & $\mathrm{w}-\mathrm{C}$ & 0.818 & w-B & 0.000 & $\mathrm{D}$ & 0.682 & $\mathrm{kl}$ & 0.00 \\
\hline Spunta & 2.000 & $\mathrm{~m}-\mathrm{r}$ & 3.091 & $b-g$ & 1.727 & $p-t$ & 0.000 & $\mathrm{D}$ & 1.705 & $\mathrm{~cd}$ & 0.00 \\
\hline Elodi & 3.273 & $b-e$ & 3.091 & $b-g$ & 0.000 & $\mathrm{D}$ & 0.000 & $\mathrm{D}$ & 1.591 & cde & 0.00 \\
\hline $97 f-25-25$ & 3.182 & $b-f$ & 2.182 & $k-p$ & 0.727 & $\mathrm{w}-\mathrm{C}$ & 0.818 & w-B & 1.727 & $\mathrm{c}$ & 25.71 \\
\hline Triomph & 2.000 & $\mathrm{~m}-\mathrm{r}$ & 2.455 & h-n & 0.000 & $\mathrm{D}$ & 0.000 & $\mathrm{D}$ & 1.114 & $g-j$ & 0.00 \\
\hline $\begin{array}{l}\text { Lady } \\
\text { Rosetta }\end{array}$ & 1.273 & S-W & 2.182 & $\mathrm{k}-\mathrm{p}$ & 3.000 & b-h & 0.545 & $y-D$ & 1.750 & $\mathrm{c}$ & 42.86 \\
\hline 95k-94 & 1.455 & $r-v$ & 2.000 & m-r & 1.273 & s-w & 0.909 & $v-A$ & 1.409 & d-g & 62.50 \\
\hline Oceania & 1.273 & S-W & 1.000 & $\mathrm{u}-\mathrm{z}$ & 2.091 & $1-q$ & 0.000 & $\mathrm{D}$ & 1.091 & hij & 0.00 \\
\hline Nicola & 1.818 & o-s & 2.364 & i-o & 1.000 & $\mathrm{u}-\mathrm{z}$ & 0.000 & $\mathrm{D}$ & 1.295 & e-h & 0.00 \\
\hline Fridor & 2.182 & $k-p$ & 1.545 & $\mathrm{q}-\mathrm{u}$ & 1.091 & $\mathrm{u}-\mathrm{y}$ & 0.000 & $\mathrm{D}$ & 1.205 & ghi & 0.00 \\
\hline Naga & 3.455 & $a b c$ & 2.818 & $d-j$ & 1.000 & $\mathrm{u}-\mathrm{z}$ & 0.000 & $\mathrm{D}$ & 1.818 & $\mathrm{c}$ & 0.00 \\
\hline Diamant & 1.909 & $n-r$ & 1.818 & o-s & 0.273 & $\mathrm{BCD}$ & 0.000 & $\mathrm{D}$ & 1.000 & hij & 0.00 \\
\hline Agria & 2.545 & g-m & 2.091 & $1-q$ & 1.545 & $\mathrm{q}-\mathrm{u}$ & 0.818 & $\mathrm{w}-\mathrm{B}$ & 1.750 & $\mathrm{c}$ & 32.14 \\
\hline 99-981 & 1.818 & o-s & 0.455 & $z-D$ & 0.000 & $\mathrm{D}$ & 0.000 & $\mathrm{D}$ & 0.568 & $\operatorname{lm}$ & 0.00 \\
\hline $97-980$ & 1.000 & $\mathrm{u}-\mathrm{z}$ & 1.727 & $p-t$ & 0.000 & $\mathrm{D}$ & 0.000 & $\mathrm{D}$ & 0.682 & $\mathrm{kl}$ & 0.00 \\
\hline $94 f$ & 1.091 & u-y & 0.727 & $\mathrm{w}-\mathrm{C}$ & 0.364 & A-D & 0.000 & $\mathrm{D}$ & 0.545 & $\operatorname{lmn}$ & 0.00 \\
\hline Jelly & 2.636 & $f-1$ & 2.545 & $\mathrm{~g}-\mathrm{m}$ & 1.000 & $\mathrm{u}-\mathrm{z}$ & 0.000 & $\mathrm{D}$ & 1.545 & $c-f$ & 0.00 \\
\hline Presto & 1.000 & $\mathrm{u}-\mathrm{z}$ & 1.727 & $\mathrm{p}-\mathrm{t}$ & 0.636 & $\mathrm{x}-\mathrm{C}$ & 0.000 & $\mathrm{D}$ & 0.841 & $\mathrm{jkl}$ & 0.00 \\
\hline Marabel & 0.818 & $\mathrm{w}-\mathrm{B}$ & 1.000 & $\mathrm{u}-\mathrm{z}$ & 1.455 & $\mathrm{r}-\mathrm{v}$ & 0.000 & $\mathrm{D}$ & 0.818 & $\mathrm{jkl}$ & 0.00 \\
\hline $97 f-267$ & 0.455 & $\mathrm{z}-\mathrm{D}$ & 0.364 & A-D & 2.364 & $\mathrm{i}-\mathrm{o}$ & 0.636 & $\mathrm{x}-\mathrm{C}$ & 0.955 & $\mathrm{ijk}$ & 140.00 \\
\hline Picasso & 3.364 & bcd & 2.545 & $g-m$ & 2.909 & $c-i$ & 1.182 & $\mathrm{t}-\mathrm{x}$ & 2.500 & $\mathrm{a}$ & 35.14 \\
\hline Provinta & 2.727 & e-k & 2.545 & $\mathrm{~g}-\mathrm{m}$ & 1.818 & o-s & 0.000 & $\mathrm{D}$ & 1.773 & $\mathrm{c}$ & 0.00 \\
\hline Arinda & 3.364 & bcd & 3.091 & $b-g$ & 0.545 & $y-D$ & 0.364 & A-D & 1.841 & $\mathrm{c}$ & 10.81 \\
\hline Bolista & 4.000 & $\mathrm{a}$ & 2.273 & $j-p$ & 2.273 & $j-p$ & 0.182 & $\mathrm{CD}$ & 2.182 & $\mathrm{~b}$ & 4.55 \\
\hline Santa & 1.091 & $\mathrm{u}-\mathrm{y}$ & 0.000 & $\mathrm{D}$ & 0.000 & $\mathrm{D}$ & 0.000 & $\mathrm{D}$ & 0.273 & $\mathrm{mn}$ & 0.00 \\
\hline Mean NaCl & 1.987 & $\mathbf{a}$ & 1.768 & b & 1.11 & c & 0.282 & d & & & \\
\hline
\end{tabular}

$* \%$ control $=$ values at the $150 \mathrm{mMNaCl}$ divided by the values at control $(0.0 \mathrm{NaCl}) \times 100$

** Means with the same letter were not significantly different $(\mathrm{P} \leq 5 \%)$. 
Effect of salinity onmicrotuberizationin vitro:

$\mathrm{NaCl}$ stress significantly affected number of microtuber/jar as an average over 29 tested genotypes (Table 3). Microtuber number was the highest (average 3.8 microtubers/jar) at $0.0 \mathrm{NaCl}$. This number decreased significantly with the increase in $\mathrm{NaCl}$ concentration in the medium, reached the least value (average 2.06 microtubers) at $150 \mathrm{mM} \mathrm{NaCl}(54.8 \%$ of the control). The average microtuber weight was also affected by $\mathrm{NaCl}$ stress (Table 4). Microtuber weight decreased significantly from $272 \mathrm{mg}$ at $0.0 \mathrm{NaCl}$, to about $150 \mathrm{mg}$ at $50-150 \mathrm{mM} \mathrm{NaCl}$, with an average $44.85 \%$ decrease compared to the control.

Potato microtuber induction and development were different among the different genotypes. As tested over all salinity levels, microtuber number was the highest in cv. Naga (7.2), followed by Bolista, Sante, Margod, Oceaniaand Lady Rosetta ( $>4.0$ microtubers/jar). The least number of microtubers was recorded on cvs. Arinda, Proventa, Fridor and Triomph (Table 3).

Table (3). Effect of $\mathrm{NaCl}$ concentration on the in vitro average microtuber number / jar in 29 potato genotypes.

\begin{tabular}{|c|c|c|c|c|c|c|c|c|c|c|c|}
\hline \multirow{3}{*}{$\begin{array}{l}\text { CV } \\
\text { Safran }\end{array}$} & \multicolumn{2}{|c|}{$\mathbf{0 . 0}$} & \multicolumn{2}{|c|}{50} & \multicolumn{2}{|c|}{100} & \multicolumn{2}{|c|}{150} & \multirow{2}{*}{\multicolumn{2}{|c|}{ Mean cv. }} & \multirow{3}{*}{$\begin{array}{c}\begin{array}{c}\% \text { of } \\
\text { control* }\end{array} \\
50.00\end{array}$} \\
\hline & \multicolumn{8}{|c|}{ Average microtuber no./jar } & & & \\
\hline & 5.200 & $\mathrm{f}-\mathrm{i}^{* *}$ & 4.600 & g-k & 3.400 & $\mathrm{u}-\mathrm{Z}$ & 2.600 & $\mathrm{~W}-\mathrm{Z}$ & 3.950 & ef & \\
\hline 94f-8101 & 5.000 & $f-j$ & 4.000 & $\mathrm{i}-\mathrm{n}$ & 2.800 & $n-t$ & 2.000 & $\mathrm{r}-\mathrm{x}$ & 3.450 & de & 40.00 \\
\hline Margod & 6.200 & def & 6.200 & def & 4.200 & $\mathrm{i}-\mathrm{m}$ & 2.200 & q-w & 4.700 & $b$ & 35.48 \\
\hline Universa & 3.800 & $\mathrm{j}-\mathrm{o}$ & 2.400 & $\mathrm{p}-\mathrm{v}$ & 1.400 & $\mathrm{u}-\mathrm{z}$ & 1.400 & $\mathrm{u}-\mathrm{z}$ & 2.250 & ghi & 36.84 \\
\hline Alaska & 3.000 & $\mathrm{~m}-\mathrm{s}$ & 2.000 & $\mathrm{r}-\mathrm{x}$ & 1.400 & $\mathrm{u}-\mathrm{z}$ & 2.400 & $\mathrm{p}-\mathrm{v}$ & 2.200 & ghi & 80.00 \\
\hline Spunta & 7.000 & $\mathrm{~cd}$ & 3.400 & $\mathrm{k}-\mathrm{q}$ & 3.000 & $\mathrm{~m}-\mathrm{s}$ & 2.200 & $q-w$ & 3.900 & $\mathrm{~cd}$ & 31.43 \\
\hline Elodi & 4.200 & $\mathrm{i}-\mathrm{m}$ & 2.400 & $\mathrm{p}-\mathrm{v}$ & 1.600 & $t-z$ & 1.200 & $\mathrm{~V}-\mathrm{z}$ & 2.350 & $\mathrm{gh}$ & 28.57 \\
\hline $97 f-25-25$ & 5.800 & d-g & 3.800 & $\mathrm{j}-\mathrm{o}$ & 1.800 & s-y & 1.400 & $\mathrm{u}-\mathrm{z}$ & 3.200 & e & 24.14 \\
\hline Triomph & 1.600 & $t-z$ & 1.400 & $\mathrm{u}-\mathrm{z}$ & 1.000 & W-Z & 0.600 & $\mathrm{yz}$ & 1.150 & $\mathrm{kl}$ & 37.50 \\
\hline $\begin{array}{l}\text { Lady } \\
\text { Rosetta }\end{array}$ & 5.800 & d-g & 4.600 & g-k & 4.000 & i-n & 2.400 & $\mathrm{p}-\mathrm{V}$ & 4.200 & $\mathrm{bc}$ & 41.38 \\
\hline 95k-94 & 4.000 & $i-n$ & 3.000 & $\mathrm{~m}-\mathrm{s}$ & 2.200 & $q-w$ & 0.800 & xyz & 2.500 & fg & 20.00 \\
\hline Oceania & 2.200 & $q-w$ & 5.600 & $e-h$ & 5.600 & e-h & 5.800 & $d-g$ & 4.800 & b & 263.64 \\
\hline Nicola & 3.000 & m-s & 1.600 & $t-z$ & 0.800 & xyz & 1.200 & $\mathrm{~V}-\mathrm{z}$ & 1.650 & ijkl & 40.00 \\
\hline Fridor & 1.600 & $t-z$ & 1.000 & $\mathrm{~W}-\mathrm{Z}$ & 1.200 & $\mathrm{~V}-\mathrm{z}$ & 1.200 & $\mathrm{~V}-\mathrm{z}$ & 1.250 & $\mathrm{jkl}$ & 75.00 \\
\hline Naga & 9.600 & $\mathrm{a}$ & 7.800 & $\mathrm{bc}$ & 6.800 & cde & 4.600 & g-k & 7.200 & $\mathrm{a}$ & 47.92 \\
\hline Diamant & 3.200 & $1-q r$ & 4.400 & h-1 & 2.600 & $\mathrm{o}-\mathrm{u}$ & 2.400 & $\mathrm{p}-\mathrm{V}$ & 3.150 & e & 75.00 \\
\hline Agria & 3.600 & $k-p$ & 1.600 & $\mathrm{t}-\mathrm{z}$ & 1.800 & s-y & 1.000 & $\mathrm{~W}-\mathrm{Z}$ & 2.000 & ghi & 27.78 \\
\hline 99-981 & 3.200 & $1-r$ & 2.600 & $\mathrm{o}-\mathrm{u}$ & 1.000 & W-Z & 0.400 & $\mathrm{z}$ & 1.800 & hij & 12.50 \\
\hline $97-980$ & 4.200 & $\mathrm{i}-\mathrm{m}$ & 1.800 & s-y & 2.000 & $r-x$ & 2.000 & $r-x$ & 2.500 & $\mathrm{fg}$ & 47.62 \\
\hline $94 f$ & 3.000 & m-s & 2.600 & $\mathrm{o}-\mathrm{u}$ & 1.600 & $t-z$ & 1.000 & $\mathrm{~W}-\mathrm{Z}$ & 2.050 & ghi & 33.33 \\
\hline Jelly & 1.400 & $\mathrm{u}-\mathrm{Z}$ & 1.200 & $\mathrm{~V}-\mathrm{z}$ & 2.000 & $r-x$ & 2.600 & o-u & 1.800 & hij & 185.71 \\
\hline Presto & 1.400 & $\mathrm{u}-\mathrm{z}$ & 3.000 & $\mathrm{~m}-\mathrm{s}$ & 1.600 & $t-z$ & 1.000 & w-Z & 1.750 & $\mathrm{~h}-\mathrm{k}$ & 71.43 \\
\hline Marabel & 4.000 & $i-n$ & 2.200 & $q-w$ & 2.000 & $r-x$ & 1.800 & s-y & 2.500 & fg & 45.00 \\
\hline $97 f-267$ & 1.400 & $\mathrm{u}-\mathrm{z}$ & 1.600 & $\mathrm{t}-\mathrm{z}$ & 2.200 & $q-w$ & 3.000 & $\mathrm{~m}-\mathrm{s}$ & 2.050 & ghi & 214.29 \\
\hline Picasso & 1.400 & $\mathrm{u}-\mathrm{z}$ & 1.000 & W-Z & 1.000 & W-Z & 3.200 & $1-r$ & 1.650 & $\mathrm{i}-1$ & 228.57 \\
\hline Provinta & 1.600 & $t-z$ & 1.000 & $\mathrm{~W}-\mathrm{Z}$ & 1.200 & $\mathrm{~V}-\mathrm{Z}$ & 1.000 & $\mathrm{~W}-\mathrm{Z}$ & 1.200 & $\mathrm{jkl}$ & 62.50 \\
\hline Arinda & 1.000 & W-Z & 1.000 & w-Z & 1.000 & w-z & 1.400 & $\mathrm{u}-\mathrm{z}$ & 1.100 & 1 & 140.00 \\
\hline Bolista & 8.800 & $a b$ & 4.000 & i-n & 3.400 & $\mathrm{k}-\mathrm{q}$ & 1.600 & $\mathrm{t}-\mathrm{z}$ & 4.450 & $\mathrm{bc}$ & 18.18 \\
\hline Sante & 3.200 & $1-r$ & 4.400 & h-1 & 4.200 & $\mathrm{i}-\mathrm{m}$ & 5.600 & $e-h$ & 4.350 & $\mathrm{bc}$ & 175.00 \\
\hline Mean $\mathrm{NaCl}$ & 3.772 & $\mathbf{a}$ & 2.972 & b & 2.37 & c & 2.069 & d & & & \\
\hline
\end{tabular}

$* \%$ control $=$ values at the $150 \mathrm{mMNaCl}$ divided by the values at control $(0.0 \mathrm{NaCl}) \times 100$

** Means with the same letter were not significantly different $(\mathrm{P} \leq 5 \%)$ 
Significant differences among potato genotypes in average microtuber weight were also detected (Table 4). The highest average microtuber weight was recorded in cv. Fridor (548 mg), followed by Lady Rosetta (460 $\mathrm{mg}$ ) and line 97 F.276 (420 mg/microtuber). The least microtuber weight was found in cvs. Nicola, 99.981, Elodi and 94F-8101.

Microtuber induction and development were significantly affected by the $\mathrm{NaCl} x$ genotype interaction. For the number of microtuber/jar (Table 3 ), the cv. Naga recorded the highest number (9.6 microtubers) on the medium at $0.0 \mathrm{NaCl}$ (control), followed by cv. Bolista ( 8.8 microtubers). At 50 and $100 \mathrm{mMNaCl}$, the cv. Naga also recorded the highest microtuber number among all other genotypes. At the highest $\mathrm{NaCl}$ concentration $(150 \mathrm{mM})$, the cv. Oceania and Sante produced larger number of microtubers. Although microtuber number/jar decrease in most genotypes with increasing salt level in the medium, the genotypes Oceania, Jelly, 97.267, Picasso, Arinda and Sante had higher number of microtubers at the highest level of $\mathrm{NaCl}$, as compared to the control, indicating different response of potato genotypes salinity stress.

Average microtuber weight was significantly affected by the salt $x$ genotype interaction (Table 4). The highest microtuber weight was recorded on cv. Fridor (ave. $1950 \mathrm{mg}$ ) at $0.0 \mathrm{NaCl}$. The cv. Lady Rosetta recorded the highest microtuber weight at 50 $\mathrm{mMNaCl}$, while the cv. Naga produced higher microtuber weight at $100 \mathrm{mM} \mathrm{NaCl}$ (ave. $498 \mathrm{mg}$ ). At the highest $\mathrm{NaCl}$ level $(150 \mathrm{mM})$, the potato line 97F.267 recorded higher microtuber weight (905 $\mathrm{mg}$ /tuber) over all other genotypes at this highest $\mathrm{NaCl}$ level, indicating clearly the different responses of potato genotypes to the different $\mathrm{NaCl}$ stress levels on microtuber weight.

Table (4). Effect of $\mathrm{NaCl}$ concentration on the in vitro average microtuber weight in 29 potato genotypes.

\begin{tabular}{|c|c|c|c|c|c|c|c|c|c|c|c|}
\hline \multirow{3}{*}{$\begin{array}{r}\text { CV } \\
\text { Safran }\end{array}$} & \multicolumn{2}{|c|}{0.0} & \multicolumn{2}{|c|}{50} & \multicolumn{2}{|c|}{100} & \multicolumn{2}{|c|}{150} & \multirow{2}{*}{\multicolumn{2}{|c|}{ Mean cv. }} & \multirow{3}{*}{$\begin{array}{c}\begin{array}{c}\% \text { of } \\
\text { control* }\end{array} \\
33.30\end{array}$} \\
\hline & \multicolumn{8}{|c|}{ Average microtuber Weight (g) } & & & \\
\hline & 0.398 & $e-j$ & 0.326 & $f-n$ & 0.153 & $q-u$ & 0.132 & $\mathrm{r}-\mathrm{u}$ & 0.252 & $\mathrm{e}-\mathrm{h}^{* *}$ & \\
\hline $94 f-8101$ & 0.723 & $\mathrm{bc}$ & 0.510 & $c-f$ & 0.077 & $\mathrm{p}-\mathrm{u}$ & 0.041 & $r-u$ & 0.338 & $\mathrm{~cd}$ & 5.66 \\
\hline Margod & 0.189 & $\mathrm{j}-\mathrm{u}$ & 0.234 & $\mathrm{i}-\mathrm{t}$ & 0.115 & $\mathrm{~m}-\mathrm{u}$ & 0.079 & $\mathrm{p}-\mathrm{u}$ & 0.154 & $g-j$ & 41.93 \\
\hline Universa & 0.144 & $\mathrm{~m}-\mathrm{u}$ & 0.128 & $\mathrm{~m}-\mathrm{u}$ & 0.089 & $\mathrm{o}-\mathrm{u}$ & 0.074 & $\mathrm{p}-\mathrm{u}$ & 0.108 & g-k & 51.43 \\
\hline Alaska & 0.081 & $\mathrm{p}-\mathrm{u}$ & 0.028 & $\mathrm{r}-\mathrm{u}$ & 0.069 & $q-u$ & 0.097 & $\mathrm{~m}-\mathrm{u}$ & 0.069 & $\mathrm{jk}$ & 119.66 \\
\hline Spunta & 0.384 & e-1 & 0.138 & $\mathrm{~m}-\mathrm{u}$ & 0.141 & $\mathrm{~m}-\mathrm{u}$ & 0.474 & $\mathrm{~d}-\mathrm{h}$ & 0.285 & cde & 123.38 \\
\hline Elodi & 0.178 & $\mathrm{j}-\mathrm{u}$ & 0.024 & stu & 0.027 & stu & 0.002 & tu & 0.058 & $\mathrm{jk}$ & 1.07 \\
\hline $97 f-25-25$ & 0.187 & $\mathrm{j}-\mathrm{u}$ & 0.149 & $\mathrm{~m}-\mathrm{u}$ & 0.023 & stu & 0.010 & tu & 0.092 & h-k & 5.46 \\
\hline Triomph & 0.072 & $\mathrm{q}-\mathrm{u}$ & 0.031 & $\mathrm{r}-\mathrm{u}$ & 0.027 & stu & 0.002 & tu & 0.033 & $\mathrm{k}$ & 2.33 \\
\hline $\begin{array}{l}\text { Lady } \\
\text { Rosetta }\end{array}$ & 0.670 & $\mathrm{~cd}$ & 0.537 & $c-f$ & 0.384 & e-1 & 0.261 & h-r & 0.463 & b & 38.87 \\
\hline $95 k-94$ & 0.142 & $\mathrm{~m}-\mathrm{u}$ & 0.070 & $q-u$ & 0.037 & $\mathrm{r}-\mathrm{u}$ & 0.020 & stu & 0.067 & jjk & 14.09 \\
\hline Oceania & 0.118 & stu & 0.155 & $1-u$ & 0.392 & e-k & 0.330 & $\mathrm{f}-\mathrm{m}$ & 0.249 & d-g & 280.40 \\
\hline Nicola & 0.044 & $q-u$ & 0.011 & tu & 0.013 & tu & 0.022 & stu & 0.023 & $\mathrm{k}$ & 50.11 \\
\hline Fridor & 1.950 & $\mathrm{a}$ & 0.247 & h-s & 0.117 & $\mathrm{~m}-\mathrm{u}$ & 0.021 & stu & 0.584 & $\mathrm{a}$ & 1.06 \\
\hline Naga & 0.524 & $c-f$ & 0.401 & $e-j$ & 0.498 & $\mathrm{c}-\mathrm{g}$ & 0.156 & $1-u$ & 0.395 & $\mathrm{bc}$ & 29.70 \\
\hline Diamant & 0.099 & $\mathrm{~m}-\mathrm{u}$ & 0.084 & o-u & 0.049 & $q-u$ & 0.009 & tu & 0.060 & $\mathrm{jk}$ & 9.54 \\
\hline Agria & 0.068 & $q-u$ & 0.128 & $\mathrm{~m}-\mathrm{u}$ & 0.036 & $\mathrm{r}-\mathrm{u}$ & 0.006 & tu & 0.059 & $\mathrm{jk}$ & 8.17 \\
\hline 99-981 & 0.094 & $\mathrm{n}-\mathrm{u}$ & 0.018 & stu & 0.031 & $\mathrm{r}-\mathrm{u}$ & 0.001 & $\mathrm{u}$ & 0.036 & $\mathrm{k}$ & 0.85 \\
\hline 97-980 & 0.178 & $j-u$ & 0.051 & $q-u$ & 0.216 & $\mathrm{j}-\mathrm{u}$ & 0.306 & $f-p$ & 0.188 & $e-h$ & 171.90 \\
\hline $94 f$ & 0.086 & o-u & 0.080 & $\mathrm{p}-\mathrm{u}$ & 0.008 & tu & 0.010 & tu & 0.046 & $\mathrm{jk}$ & 11.41 \\
\hline Jelly & 0.127 & stu & 0.125 & stu & 0.386 & e-1 & 0.277 & $g-q$ & 0.229 & $e-i$ & 218.55 \\
\hline Presto & 0.030 & $\mathrm{r}-\mathrm{u}$ & 0.093 & $\mathrm{n}-\mathrm{u}$ & 0.059 & $q-u$ & 0.044 & $q-u$ & 0.057 & $\mathrm{jk}$ & 149.87 \\
\hline Marabel & 0.194 & $j-u$ & 0.122 & $\mathrm{~m}-\mathrm{u}$ & 0.071 & $q-u$ & 0.023 & stu & 0.103 & h-k & 12.03 \\
\hline $97 f-267$ & 0.313 & tu & 0.225 & stu & 0.250 & $\mathrm{q}-\mathrm{u}$ & 0.905 & $\mathrm{~b}$ & 0.423 & def & 289.19 \\
\hline Picasso & 0.229 & $\mathrm{r}-\mathrm{u}$ & 0.276 & g-q & 0.330 & $\mathrm{f}-\mathrm{m}$ & 0.329 & $\mathrm{f}-\mathrm{m}$ & 0.291 & def & 143.58 \\
\hline Provinta & 0.164 & $\mathrm{k}-\mathrm{u}$ & 0.047 & $\mathrm{q}-\mathrm{u}$ & 0.026 & stu & 0.004 & tu & 0.060 & $\mathrm{jk}$ & 2.52 \\
\hline Arinda & 0.031 & $r-u$ & 0.090 & $\mathrm{o}-\mathrm{u}$ & 0.145 & $\mathrm{~m}-\mathrm{u}$ & 0.086 & $\mathrm{o}-\mathrm{u}$ & 0.088 & h-k & 277.82 \\
\hline Bolista & 0.315 & f-o & 0.142 & $\mathrm{~m}-\mathrm{u}$ & 0.123 & $\mathrm{~m}-\mathrm{u}$ & 0.042 & $\mathrm{r}-\mathrm{u}$ & 0.155 & $f-j$ & 13.37 \\
\hline Sante & 0.157 & $\mathrm{q}-\mathrm{u}$ & 0.178 & $\mathrm{j}-\mathrm{u}$ & 0.463 & $\mathrm{~d}-\mathrm{i}$ & 0.581 & cde & 0.345 & $\mathrm{~cd}$ & 369.89 \\
\hline Mean $\mathrm{NaCl}$ & 0.272 & $\mathbf{a}$ & 0.160 & b & 0.15 & b & 0.150 & b & & & \\
\hline
\end{tabular}

$* \%$ control $=$ values at the $150 \mathrm{mMNaCl}$ divided by the values at control $(0.0 \mathrm{NaCl}) \times 100$

** Means with the same letter were not significantly different $(\mathrm{P} \leq 5 \%)$. 
Results of potato genotype screening for salinity stress: Based on plantlet length at the highest salinity level as percent of control, potato genotypes could be ranked as follow (from Table 1): Agria $>$ Arinda $>97 \mathrm{~F}-$ $267>$ Presto $>$ Univrsa $>$ Oceania $>97$ f.25-25 $>97-980$ $>$ 95K-94 $>$ Bolista $>$ Marabel $>$ Sante $>$ Safran $>$ 99$981>$ Fridor $>$ Proventa $>$ Spunta $>$ Alaska $>$ Nicloa $>$ Picasso $>$ 94F-8101 $>$ Triomph $>$ Jelly $>$ Naga $>$ Diamont $>$ Margod $>$ Lady Rosetta $>94 \mathrm{~F}>$ Elodi.

Based on the relative number of microtuber formed at the highest salinity level as percent of control (Table 3 ), potato genotypes are ranked as follow: Oceania $>$ Picasso $>$ 97F-267 $>$ Jelly $>$ Sante $>$ Arinda $>$ Alaska $>$ Fridor $=$ Diamont $>$ Presto $>$ Proventa $>$ Safran $>$ Naga $>$ 97-980 > Marabel $>$ 94F-8101 > Lady Rosetta $>$ Nicloa $>$ Triomph $>$ Universa $>$ Margod $>94 \mathrm{~F}>$ Spunta $>$ Elodi $>$ Agria $>$ 97f-25-25 $>$ 95K-94 $>$ Bolista $>99-981$.

\section{Photosynthetic pigment contents under salt stress:}

The accumulation of all pigments as chlorophyll a (chl. a), chlorophyll b (chl. b), total (chl. a+b) and caratenoids under $100 \mathrm{mM} \mathrm{NaCl}$ indicated significant decline over the control $(0.0 \mathrm{NaCl})$ as shown in Table (5). Chlorophyll a, chlorophyll b, total chl and carotenoids contents represented $77.0 \%, 85.7 \% 80.7 \%$ and $75.7 \%$ of the control, respectively.
As an average over the tested levels of $\mathrm{NaCl}$, potato genotypes showed significant differences in their pigment contents (Table 5). For chl. a, the cv. Bolista, followed by Safran recorded the highest content, while the lowest chl. a was found in cv. Nicola. Chlorophyll b was also the highest in cv. Bolista and the lowest in cv. Nicola, and the same trend was found in total chl. Significant differences among potato cvs were also detected in their carotenoids content, with cv. Universa being the highest, and cvsDiamant and Agria had the least. Other cvs had almost the same carotenoid contents.

The interaction effect was found significant for all analyzed pigment contents. All genotypes showed less chl. a at $100 \mathrm{mM} \mathrm{NaCl}$ than the control (Table 5), but in different degree. As percent of control, chl. a contents were $78.6,82.9,91.1,73.8,81.8,60.3$, and $73 \%$ of the controls treatment for the cvs Safran, Universa, Triomph, Nicola, Diamant, Agria, and Bolista, respectively. Chl. b and total chl also followed the same trend. Carotenoids were significantly decreased under $\mathrm{NaCl}$ stress in all cvs, except Universa. The carotenoids contents as $\%$ of control were $86.3 \%, 53.9 \% 88.3 \%$ $50.3 \% 67.1 \%$ and $73.5 \%$ in the cvs Safran, Triomph, Nicola, Diamant, Agria and Bolista, respectively.

Table (5). Effect of in vitro $\mathrm{NaCl}$ stress on chlorophyll and carotenoid contents in 7 potato genotypes.

\begin{tabular}{|c|c|c|c|c|c|c|c|c|}
\hline \multirow{2}{*}{$\begin{array}{l}\mathrm{CV} \\
\text { Sofron }\end{array}$} & \multirow{2}{*}{$\frac{\mathrm{NaCl}}{\text { Control }}$} & \multicolumn{2}{|c|}{ Chl.a } & \multicolumn{2}{|c|}{ Chl.b } & \multirow{2}{*}{$\frac{\text { Total Chl. }}{56.26}$} & \multicolumn{2}{|c|}{ Carotenoids } \\
\hline & & $35.15 *$ & $\mathrm{~b}$ & 21.12 & g & & 13.37 & e \\
\hline & $100 \mathrm{mM}$ & 27.65 & ef & 15.1 & e & 42.75 & 11.55 & $\mathrm{f}$ \\
\hline \multirow{4}{*}{ Universa } & Mean CV & 31.4 & $\mathrm{~b}$ & 18.11 & $\mathrm{~d}$ & 49.5 & 12.46 & bc \\
\hline & Control & 29.84 & $\mathrm{~d}$ & 23.36 & $\mathrm{~cd}$ & 53.19 & 16.51 & $\mathrm{bc}$ \\
\hline & $100 \mathrm{mM}$ & 24.76 & $\mathrm{~g}$ & 21.83 & cde & 46.59 & 18.02 & $\mathrm{~b}$ \\
\hline & Mean CV & 27.3 & $\mathrm{~cd}$ & 22.6 & $b$ & 49.9 & 17.26 & $\mathrm{a}$ \\
\hline \multirow{3}{*}{ Triomph } & Control & 29.84 & $\mathrm{~d}$ & 23.36 & $\mathrm{~cd}$ & 53.19 & 16.51 & bc \\
\hline & $100 \mathrm{mM}$ & 27.17 & $\mathrm{f}$ & 21.72 & de & 51.88 & 8.9 & $\mathrm{~g}$ \\
\hline & Mean CV & 28.51 & $\mathrm{~cd}$ & 22.54 & $\mathrm{~b}$ & 51.05 & 12.7 & $\mathrm{~b}$ \\
\hline \multirow{2}{*}{ Nicola } & Control & 21.34 & $\mathrm{~h}$ & 15.95 & $\mathrm{~g}$ & 37.27 & 9.39 & $\mathrm{~g}$ \\
\hline & $100 \mathrm{mM}$ & 15.77 & $\mathrm{i}$ & 13.28 & $\mathrm{~h}$ & 29.04 & 8.29 & $\mathrm{~g}$ \\
\hline \multirow{4}{*}{ Diamant } & Mean CV & 18.56 & ef & 14.61 & e & 33.17 & 8.84 & $\mathrm{~d}$ \\
\hline & Control & 28.91 & de & 19.23 & $\mathrm{f}$ & 48.12 & 11.45 & $\mathrm{f}$ \\
\hline & $100 \mathrm{mM}$ & 23.67 & $\mathrm{~g}$ & 18.77 & $\mathrm{f}$ & 42.44 & 5.76 & $\mathrm{~h}$ \\
\hline & Mean CV & 26.29 & $\mathrm{~d}$ & 19 & $\mathrm{~cd}$ & 45.29 & 8.6 & $\mathrm{~d}$ \\
\hline \multirow{2}{*}{ Agria } & Control & 33.27 & $\mathrm{~cd}$ & 23.47 & $\mathrm{c}$ & 56.72 & 14.12 & de \\
\hline & $100 \mathrm{mM}$ & 20.08 & $\mathrm{~h}$ & 17.79 & $\mathrm{f}$ & 37.85 & 9.47 & $\mathrm{~g}$ \\
\hline \multirow{4}{*}{ Bolista } & Mean CV & 26.68 & $\mathrm{~d}$ & 20.63 & bc & 47.3 & 11.7 & $\mathrm{c}$ \\
\hline & Control & 41.58 & $\mathrm{a}$ & 35.99 & $\mathrm{a}$ & 77.55 & 20.66 & $\mathrm{a}$ \\
\hline & $100 \mathrm{mM}$ & 30.35 & $\mathrm{~d}$ & 30.76 & $\mathrm{~b}$ & 61.11 & 15.22 & $\mathrm{~cd}$ \\
\hline & Mean CV & 35.96 & $\mathrm{a}$ & 33.38 & $\mathrm{a}$ & 69.33 & 12.94 & $\mathrm{a}$ \\
\hline Mean of control & & 31.42 & $\mathrm{a}$ & 23.21 & $\mathrm{a}$ & & 14.57 & $\bar{a}$ \\
\hline Mean of $\mathrm{NaCl}$ & & 24.21 & $\mathrm{~b}$ & 19.89 & $\mathrm{~b}$ & & 11.03 & $\mathrm{~b}$ \\
\hline
\end{tabular}

* Means with the same letters are not significantly different at $\mathrm{p} \leq 5 \%$. 
Free amino acids, proline and antioxidant enzyme analysis under salt stress:

Salt stress resulted in increased proline content (124.1\% of the control), while SOD activity was not significantly different than the control. Total amino acids were also higher under salt stress $(139.6 \%)$ than the control, while CAT activity was lower $(42.5 \%$ of the control) as shown in Table (6).

Potato tissue differed significantly in free amino acids, proline, SOD, and CAT profiles (Table 6). The cvs. Diamant, Agria and Bolista had higher amino acids contents than cvs Safran, Universa, Triomph and Nicola as tested over the two salinity treatments. The cv. Bolista had the highest proline followed by Agria, Nicola and Safran, while cvs Triomph had the lowest proline content. The cvs Universa and Diamant did not differ significantly in proline content. The activity of SOD was highest in cv. Safran, followed by Universa, Triomph and Agria, whereas the cv. Bolista recorded the least SOD. The activity of CAT enzyme was the highest in cv. Agria and Bolista, and the lowest in cvs Safran and Universa.

The $\mathrm{NaCl}$ concentration $\times$ potato genotype interactions were significant in some biochemical's examined (Table 6). Total amino acids was the highest in cvs Safran and Diamant at $100 \mathrm{mM} \mathrm{NaCl}$, while the cv. Agria and Bolista had equal free amino acids content at both 0.0 and $100 \mathrm{mM} \mathrm{NaCl}$.

Regarding proline contents, the highest recorded amount was found in shoot tissues of cv. Bolista at 0.0 and $100 \mathrm{mM} \mathrm{NaCl}$. Proline increased in all cvs under salt stress, except in cv. Agria which was decreased significantly under stress in comparison with control. Proline content was not significantly differed between 0.0 and $100 \mathrm{mM} \mathrm{NaCl}$ in cv. Bolista (Table 6).

With respect to SOD enzyme activity, the highest was found in cv. Safran under 0.0 and $100 \mathrm{mM} \mathrm{NaCl}$, followed by Universa, Triomph and Agria at the control treatment. The least SOD activity was found on cv. Bolista at $0.0 \mathrm{mM} \mathrm{NaCl}$. In general, SOD activities were lower at salt stress treatment than the control in cvs Safran, Universa, Triomph and Nicola, while the reverse was true in cvs Bolista, Agria and Diamant, where SOD activities were higher under salt stress (Table 6).

Regarding the interaction effect on CAT activity, the highest was found in cv. Bolista followed by cv. Agria under $0.0 \mathrm{NaCl}$, while the lowest CAT activities were recorded in cvs. Universa and Triomph, also under the control treatment. All cvs, except Agria and Bolista had more CAT activities under $100 \mathrm{mM} \mathrm{NaCl}$ than the control.

Table (6). Free amino acids, proline and antioxidant profile analysis of seven potato genotypes under high level of $\mathrm{NaCl}$ concentration in vitro.

\begin{tabular}{|c|c|c|c|c|c|c|c|c|c|}
\hline \multirow{3}{*}{$\begin{array}{l}\text { CV } \\
\text { Safran }\end{array}$} & \multirow{2}{*}{$\begin{array}{c}\text { NaCl } \\
\text { Control }\end{array}$} & \multicolumn{2}{|c|}{$\begin{array}{c}\text { Total free amino } \\
\text { acids (ug alanine/g } \\
\text { FW) }\end{array}$} & \multicolumn{2}{|c|}{$\begin{array}{c}\text { Proline (ug/g } \\
\text { FW) }\end{array}$} & \multicolumn{2}{|c|}{$\begin{array}{c}\text { Superoxide } \\
\text { dismutase } \\
\text { (u/gm fresh weight) }\end{array}$} & \multicolumn{2}{|c|}{$\begin{array}{c}\text { Catalase } \\
\text { (u/gm fresh } \\
\text { weight) }\end{array}$} \\
\hline & & 909.00 & $\mathrm{f}$ & 30.87 & ef & 6947.00 & $\mathrm{a}$ & 1.37 & $\mathrm{f}$ \\
\hline & $100 \mathrm{mM}$ & 3920.00 & $\mathrm{a}$ & 112.67 & $\mathrm{bc}$ & 6737.33 & $a b$ & 1.58 & def \\
\hline \multirow{4}{*}{ Universa } & Mean CV & 2414.50 & $\mathrm{c}$ & 71.77 & $\mathrm{c}$ & 6842.17 & $\mathrm{a}$ & 1.48 & $\mathrm{bc}$ \\
\hline & Control & 772.67 & $\mathrm{fg}$ & 39.20 & e & 6633.00 & $\mathrm{~b}$ & 1.18 & $\mathrm{f}$ \\
\hline & $100 \mathrm{mM}$ & 2108.67 & $\mathrm{e}$ & 68.33 & d & 6381.67 & $\mathrm{c}$ & 2.04 & d \\
\hline & Mean CV & 1440.67 & $\mathrm{~d}$ & 53.77 & $\mathrm{~d}$ & 6507.33 & $\mathrm{~b}$ & 1.61 & $\mathrm{bc}$ \\
\hline \multirow{3}{*}{ Triomph } & Control & 624.00 & $\mathrm{~g}$ & 26.63 & $\mathrm{f}$ & 6657.33 & $\mathrm{~b}$ & 1.14 & $\mathrm{f}$ \\
\hline & $100 \mathrm{mM}$ & 2218.67 & $\mathrm{e}$ & 30.33 & ef & 6576.67 & $\mathrm{bc}$ & 1.18 & $\mathrm{f}$ \\
\hline & Mean CV & 1421.33 & $\mathrm{~d}$ & 28.48 & $\mathrm{e}$ & 6617.00 & $\mathrm{~b}$ & 1.16 & $\mathrm{c}$ \\
\hline \multirow{3}{*}{ Nicola } & Control & 2596.00 & $\mathrm{~d}$ & 66.67 & $\mathrm{~d}$ & 5983.00 & $\mathrm{~d}$ & 1.51 & ef \\
\hline & $100 \mathrm{mM}$ & 2876.00 & $\mathrm{c}$ & 110.00 & $\mathrm{c}$ & 5787.67 & de & 2.09 & d \\
\hline & Mean CV & 2736.00 & $\mathrm{~b}$ & 88.33 & $\mathrm{~b}$ & 5885.33 & $\mathrm{c}$ & 1.80 & $\mathrm{bc}$ \\
\hline \multirow{3}{*}{ Diament } & Control & 3507.67 & $\mathrm{~b}$ & 34.97 & ef & 5617.00 & $\mathrm{e}$ & 2.01 & de \\
\hline & $100 \mathrm{mM}$ & 3887.00 & $\mathrm{a}$ & 66.00 & d & 5672.00 & $\mathrm{e}$ & 2.04 & $d$ \\
\hline & Mean CV & 3697.33 & $\mathrm{a}$ & 50.48 & $\mathrm{~d}$ & 5644.50 & $\mathrm{~d}$ & 2.02 & $\mathrm{~b}$ \\
\hline \multirow{2}{*}{ Agria } & Control & 3870.33 & $\mathrm{a}$ & 122.67 & $\mathrm{~b}$ & 6610.00 & $\mathrm{~b}$ & 13.52 & $\mathrm{~b}$ \\
\hline & $100 \mathrm{mM}$ & 3833.00 & $\mathrm{a}$ & 66.33 & $\mathrm{~d}$ & 6643.33 & $\mathrm{~b}$ & 5.80 & $\mathrm{c}$ \\
\hline \multirow{4}{*}{ Bolista } & Mean CV & 3851.67 & $\mathrm{a}$ & 94.50 & $\mathrm{~b}$ & 6626.67 & $\mathrm{~b}$ & 9.66 & $\mathrm{a}$ \\
\hline & Control & 3932.33 & $\mathrm{a}$ & 197.33 & $\mathrm{a}$ & 4645.33 & $\mathrm{~g}$ & 17.29 & $\mathrm{a}$ \\
\hline & $100 \mathrm{mM}$ & 3792.67 & $\mathrm{a}$ & 189.33 & $\mathrm{a}$ & 4970.33 & $\mathrm{f}$ & 1.43 & $\mathrm{f}$ \\
\hline & Mean CV & 3862.50 & $\mathrm{a}$ & 193.33 & $\mathrm{a}$ & 4807.83 & $\mathrm{e}$ & 9.36 & $\mathrm{a}$ \\
\hline \multicolumn{2}{|c|}{ Mean of control } & 2316.00 & $\mathrm{~b}$ & 74.05 & $\mathrm{~b}$ & 6156.10 & $\mathrm{a}$ & 5.43 & $\mathrm{a}$ \\
\hline \multicolumn{2}{|c|}{ Mean of $100 \mathrm{Mm} \mathrm{NaCl}$} & 3233.71 & $\mathrm{a}$ & 91.86 & $\mathrm{a}$ & 6109.86 & $\mathrm{a}$ & 2.31 & $\mathrm{~b}$ \\
\hline
\end{tabular}

* Means with the same letters are not significantly different at $\mathrm{p} \leq 5 \%$. 


\section{DISCUSSION}

General decline in growth parameters of potato plantlets, including plantlet length, and rooting with the increase of $\mathrm{NaCl}$ in the tissue culture medium. At the highest salinity level $(150 \mathrm{mM} \mathrm{NaCl})$, plantlet length and roots exhibited the largest decreases (77\% and $86 \%$ decline compared to the control, respectively). The observed reduction in growth parameters under increasing level of $\mathrm{NaCl}$ could be the results of $\mathrm{NaCl}$ induced alteration in cell expansion and cell growth, as well as enzymes activities as cited by Silva et al. (2001). Recent report showed evidence for dormant (quiescent) state in the epidermal cell layers of roots under salinity stress, associated with changes in ABA or GA biosynthesis, resulting in root growth restriction (Duan et al., 2013). Our results also indicated signification decline in photosynthetic pigment contents under high salinity level, which may lead to decreased net assimilation rate in micro shoots. It was concluded by Cui et al. (2007) that reductions in chlorophyll were the main reason affecting plantlet growth in vitro. Our results are in accordance with those of Daneshmand et al. (2010) and Khenifi et al. (2011).

Results also showed general reduction in microtuber induction and development at the highest salinity levels (100-150 $\mathrm{mM} \mathrm{NaCl}$ ) compared to the control, in varying degree based on the genotype response. Salinity negatively affected microtuber (number/jar) more than development (weight). The finding that salt stress induced restriction in microtuberization may be attributed to several factors. Firstly, it is well established that soluble sugars (sucrose) at high concentration in the medium serve as a signal for microtuber formation (Donnelly et al., 2003) with or without the addition of PGRs. It is possible that under salinity stress, translocation of sugars, or biosynthesis of hormones responsible for microtuber induction might be restricted. Secondly, the increase in salt concentration in the medium resulted in reduction in plantlet growth and rooting capacities, which may lead to decreased nutrient uptake from the medium into potential site of microtuber formation (Dobranszki et al., 2005).

The observed decreases in microtuberization under salt stress are in agreement with the results of Zhang and Donnelly (1997) and Zhang et al. (2005). Under the conditions of this study, significant differences among potato genotypes were found, in response to in vitro salt stress. These differences were expected due to the different genetic back ground, breeding origin, and maturity group of the examined genotypes. In harmony with our results, several reports demonstrated genotypic differences in salt tolerance in potato (Kharis et al., 1998; Zhang et al., 2005; Sudhersan et al., 2012).

In most of the previous reports, the in vitro screening of potato genotypes for salt stress was conducted in limited number of potato varieties, and bioassays were based on either plantlet growth from single node segments (Rahman et al., 2008) or microtuber formation under salt stress (Zhang et al., 2005). In our study, it was possible to examine 29 potato cultivars and breeding clones, most of them are recently introduced to the Egyptian growers.
Screening of these genotypes was based on both growth and tuberization. Zhang and Donnelly (1997) found that the three bioassays (single node, root tip culture, and microtuberization) under in vitro salt stress showed similar results in ranking the salinity tolerance of seven potato cultivar. However, in our results, this was not always the case. For example, the cv. Agria was found tolerant to salt stress based on plantlet height and rooting performance, but was ranked as sensitive cv. based on its reduced microtuberization under salt stress, while the cv. Oceania was ranked as tolerant to salt based on both plantlet growth and tuberization.

High salt concentration in the tissue culture medium allowed for rapid differentiation between salt tolerant and salt sensitive potato genotypes in our study, similar to previous reports (Shaterian et al., 2008). Among the examined genotypes, Diamant, Agria, Spunta and Lady Rosetta were the only genotypes previously studied for salt tolerance in vitro. In general, Oceania, 97f-267, Safran and Universa could be ranked as tolerant, while Agria, Diamant and Spunta as moderately tolerant, and Lady Rosetta, Bolista, Triomph, Elodi and 99-981 as salt sensitive genotypes. Diamant was ranked as moderately tolerant by Khenifi et al. (2011), and Spunta as more salt tolerant than Diamant (Bassam, 2007).

However, Arvin and Donnelly (2008) ranked Agria among salt tolerant genotypes based on electrolyte leakage from detached leaves of in vitro plantlet. In other study, Rahname and Ebrahimzadeh (2004) classified Agria as relatively salt tolerant, and Diamant as relatively salt sensitive. Under field condition, Mahmoud (2012) ranked potato cultivars in the other Diamant $>$ Lady Rosetta, while Spunta was the least tolerant under irrigation with salinity water. These findings are almost similar to our results.

Significant changes in photosynthetic pigment contents were detected under salt stress. As tested over the 7 examined cvs.chl a, chl b and carotenoides were decreased by $23 \%, 14.3 \%$ and $24.6 \%$ compared to the control, respectively. These finding, are in agreement with those of Li et al. (2006) and Cui et al. (2007).

Cultivar-dependent variations in photosynthetic pigments under salinity stress were detected. The genotypes Triomph, Diamant and Universa exhibited the least reduction in total chlorophyll (2.5-12\%), while most reduction was in cvs. Agria, Bolista and Safran. In salt tolerant species, photosynthetic pigment contents were shown to be higher than in tissues of salt sensitive ones (Daneshmand et al., 2010).

This may be true for the cv. Universa (salt tolerant), but not with cvs. Triomph and Diamant (salt sensitive cvs.), indicating that enhanced chlorophyll content does not necessarily be associated with salt stress tolerance. Carotenoids contents in cv. Universa was higher under salt stress than the control (109\% of the control). This increase in carotenoides in salt tolerant genotypes could protect them against salinity stress through quenching single oxygen, and thereby avoiding lipid peroxidation, and consequent oxidative damage, according to Juan et al. (2005).

Results of biochemical analysis indicated significant increase in proline and total free amino acids under salt stress, in agreement with the results of Daneshmand et 
al. (2010), Bouaziz et al. (2012) and Marcek et al. (2014). The increase in amino acids in potato plantlet under salt stress was also reported by Asensi-Fabado et al. (2014).

It was early reported that proline may be useful as salt - injury sensor in leaf and calli from leaf (PerezAlfocea et al., 1994) and may provide some means of protection against salt stress (Prasad and Potluri, 1996), or as biochemical marker for increased salt tolerance in potato (Martinez et al., 1996). Positive turger was also suggested to be maintained by osmotic adjustment to salinity stress via accumulation of proline (Heuer and Nadler, 1998). The reduced growth and cell size was shown to be possible cause of increasing proline content under salt stress (Rahnama and Ebrahimzadeh, 2004).

In the seven potato genotypes tested, five cvs. showed increase in proline at high $\mathrm{NaCl}$ level (Safran, Universa, Triomph, Nicola and Diamant) while the two cvs. Agria and Bolista had lower proline than the control. Because these genotypes were classified as salt tolerant (Agria) or sensitive (Bolista), it is not possible to draw a clear relationship between their salt tolerance and the accumulation of proline in their plantlets.

On the other hand, the assumed salt tolerant cv. Universa, or moderately salt tolerant cv. Safran had almost double the proline contents under salinity stress compared to the control, indicating positive correlation between their salt tolerances and the accumulation of proline in their tissues. This finding is in harmony with the results of Daneshmand et al. (2010). However, the report of Velasquez et al. (2005) indicated no association between the salt tolerance rating and changes in proline content. In addition, the results of Feitosa et al. (2001) showed that proline content was always higher in sensitive cvs. compared to the tolerant ones. Thus, proline accumulation and salt stress tolerance could not necessarily be linked in potato plants.

Our results also revealed significant decline in the activity of CAT enzyme under salt stress, while SOD was not changed, compared to the control. In line with these results, Marcek et al. (2014) did not find changes in SOD and CAT activities under salt stress. In contrast, the results of Celik and Atak (2012) in tobacco tissue culture indicated increase in SOD and decrease in CAT under high level of $\mathrm{NaCl}$.

In our results, SOD was higher in tissues of cv. Diamant, Agria and Bolista under salinity stress than the control, while the reverse was true in cvs. Safran, Universa, Triomph and Nicola. The cvs.Agria and Bolista are ranked as salt tolerant and salt sensitive, respectively, thus showing different tolerance groups. However, they share common increase in SOD activity under salt stress. Therefore, SOD might not be possible biochemical indicator for salinity tolerance, in contrast with previous reports (Yu-Jie et al., 2013; Sajid and Faheem, 2014).

Differences among potato genotypes in their CAT activity under salt stress were detected. It was higher under $100 \mathrm{mMNaCl}$ than the control in the cvs. Safran, Universa, Triomph, Nicola and Diamant, but was severely decreased in cvs. Agria and Bolista.These two cvs. were ranked as salt sensitive based on their reduction in microtuberization under salt stress, while the cvs. Safran and Diamant were among the tolerant genotypes, based on the same criteria. Therefore, it is possible that the tolerance to salt in the later cvs. correlates positively with their increased CAT activity, more than the former cvs. (Agria and Bolista). Antioxidant enzymes have an important role in plant defense system against oxidative stress, and some of the antioxidant enzymes have more protection role than the others, as suggested by Rahnama et al. (2003). It was also reported by M'Hamadi et al. (2009) that CAT contributes to salinity tolerance in potato.

\section{REFERENCES}

Aebi, H. (1984). Catalase in vitro. Methods in Enzymology, 105: 121.

Ahmed, K.U. and M.M. Rashid (1990). Erradication of potato virus $\times(\mathrm{pvx})$ by thermotherapy and meristem tip culture.Bangladesh Journal of Plant Pathology, 5: 65-69.

Arvin M.J., and D.J. Donnelly (2008). Screening potato cultivars and wild species to abiotic stresses using an electrolyte leakage bioassay. Journal of Agriculture Science and Technology, 10: 33-42.

Asensi-Fabado, M.A., A. Ammon, U. Sonnewald, S. Munne-Bosch and L.M. Voll (2014). Tocopherol deficiency reduces sucrose export from saltstressed potato leaves independently of oxidative stress and symplastic obstruction by callose. Journal of Experimental Botany, 10 (1): 1093.

Bassam, A. (2007). In vitro induction, isolation and selection of potato mutants tolerant to salinity. Advances in Horticultural Science, 20: 127-132.

Bouaziz, D., J. Pirrello, H. Ben Amor, A. Hammami, M. Charfeddine, A. Dhieb, M. Bouzayen and R. Gargouri-Bouzid (2012). Ectopic expression of dehydration responsive element binding proteins (StDREB2) confers higher tolerance to salt stress in potato. Plant Physiology and Biochemistry, 60: 98-108.

Celik, O. and C. Atak (2012). The effect of salt stress on antioxidant enzymes and proline contents of two Turkish tobacco varieties. Turkish Journal of Biology, 36(6): 339-356.

Christianson, M. (1982). World Environmental Limitations to Food and fiber Culture. In: Breeding Plants for less favorable environment (eds): Lewis,C.F., John Wily and N.Y. Sons., pp $1-12$.

Cui, Y., J. Zhang, X. Li, D. Wang, P. Huang, L. Wang and X. Du (2007). Physiological responses of potato plantlets in vitro to salt stress. Chinese Potato Journal, 21(1): 1-5.

Daneshmand, F. A., M.K. Javad and K. Manouchehri (2010). Physiological responses to $\mathrm{NaCl}$ stress in three wild species of potato in vitro. Acta Physiologiae Plantarum, 32(1): 91-101.

Dobranszki, J., K. Magyar-Tabori, I. Hudak (2005). Effect of nitrogen supply and dry length on in vitro tuberization. $16^{\text {th }}$ Triennial conference of the European Association for Potato Research, Jul., 17-22, Bilbao, Spain, pp. 793-796. 
Donnelly, D.J., W.K. Coleman, S.E. Coleman (2003). Potato microtuber production and performance: A review. American Journal of Potato Research, 80: 103-115.

Duan, L., D. Dietrich, CH. Ng, P.M. Chan, R. Bhalerao, M.J. Bennett, J R. Dinneny (2013). Endodermal ABA signaling promotes lateral root quiescence during salt stress in Arabidopsis seedlings. Plant Cell, 25(1): 324-341.

FAO (2012). Statistical database FAOSTAT. Available on line at: http//faostate.Fao.Org/site 567/default.Aspxx\#ancor.

Feitosa, D.L., J. Cambraia, M.A.O. Cano and H.A. Ruiz (2001). Plant growth and solute accumulation and distribution in two sorghum genotypes, under $\mathrm{NaCl}$ stress. Brazilian Journal of Plant Physiology, 13: 270-284.

Gadallah, M.A.A. (1996). Abscisic acid, temperature and salinity interactions on growth and some mineral elements in Carthamus plants. Plant Growth Regulation, 225-236.

Heuer, B. and A. Nadler (1998). Physiological response of potato plants to soil salinity and water deficit. Plant Science, 137(1): 45-51.

Juan, M., R.M. Rivero, L. Romero and J.M. Ruiz (2005). Evaluation of some nutritional and biochemical indicators in selecting salt-resistant tomato cultivars. Environmental and Experimental Botany, 54: 193-201.

Katerji, N., A. Hamdy, I.W. van Hoorn and M. Mastrorilli (2002). Mediterranean crop responses to water and soil salinity: eco-physiological and agronomic analyses. Bari: CIHEAM/IAMB Options Méditerranéennes: Serie B. Étudeset Recherches, 36: 231-249.

Khenifi, M.L., M. Boudjeniba and A. Kameli (2011). Effects of salt stress on micro propagation of potato (Solanum tuberosum L.). African Journal of Biotechnology, 10: 7840-7845.

Khrais, T., Y. Leclerc and D. Donnelly (1998). Relative salinity tolerance of potato cultivars assessed by in vitro screening. American Journal of Potato Research, 75: 207-210.

Lee, Y.P. and T. Takabashi (1966). An improved colorimetric determination of amino acids with the use of ninhydrin. Analytical Biochemistry, 14: 71-77.

Lichtenthaler, H.K. (1987). Chlorophylls and carotenoids: Pigments of photosynthetic biomembranes. Methods in Enzymology, 148: 350-382.

Li, H., Z. Zhang, L. Xu, W. Song and W. Zhou (2006). Effect of salinity on chlorophyll contents, proline accumulation and antioxidant enzyme activities of plantlets in vitro in potato. Journal of Zhejiang University (Agriculture and Life Sciences), 32: 300-306.

M'Hamdi, T. Bettaieb, Y. Harbaoui, A.A. Mougou and P.D. Jardin (2009). Insight into the role of catalases in salt stress in potato (Solanum tuberosum L.). Biotechnology, Agronomic, Society et Environment, 13(3): 373-379.
Mahmoud, M.S.M. (2012). Differential Response of some Potato varieties to irrigation with saline water. Annals of Agriculture Science, Moshtohor, 50: 327-340.

Marcek, T., M. Tkalec, Z. Vidakovic-Cifrek, M. Jezic and M. Curkovic-Perica (2014). Effect of $\mathrm{NaCl}$ stress on dihaploid Tobacco lines tolerant to Potato virus $Y$. Acta Physiologiae Plantarum, 36(7): 1739-1747.

Martinez, C.A., M. Maestri and E.G. Lani (1996). In vitro salt tolerance and proline accumulation in Andean potato (Solanum spp.) differing in frost resistance. Plant Science, 116: 177-184.

Morpurgo, R. (1991). Correlation between potato clones grown in vivo and in vitro under Sodium Chloride Stress Conditions. Plant Breeding, 107: 80-82.

Murashige, T. and F. Skoog (1962). A revised medium for rapid growth and bioassays with tobacco tissue culture. Physiologia Plantarum, 15: 473497.

Ni, X., S.S. Quisenberry, T. Heng-Moss, J. Markwell, G. Sarath, R. Klucas and F. Baxendale (2001). Oxidative responses of resistant and susceptible cereal leaves to symptomatic and nonsymptomatic cereal aphid (Hemiptera: Aphididae) feeding. Journal of Economic Entomology, 94: 743-751.

Nishikimi, M., N.A. Roa and K. Yogi (1972). Measurement of Superoxide dismutase. Biochemical and Biophysical Research Communications, 46: 849-854.

Perez-Alfocea, F., A. Santa-Cruz, G. Guerrier and M.C. Bolarin (1994). $\mathrm{NaCl}$ stress-induced organic solute changes on leaves and Calli of Lycopersicon esculentum L. pennellii and their interspecific hybrid. Journal of Plant Physiology, 143: 106-111.

Potluri, S.D.P. and P.V.D. Prasad (1993). Influence of salinity on axillary bud cultures of six lowland tropical varieties of potato (Solanum tuberosum). Plant Cell, Tissue and Organ Culture, 32: 185191.

Prasad, P.V.D., D.P. Potluri (1996). Influence of proline and hydroxyproline on salt-stressed axillary bud cultures of two varieties of potato (Solanum tuberosum). In Vitro Cellular \& Developmental Biology - Plant Journal, 32: 47-50.

Rahnama, H. and H. Ebrahimzadeh (2004). The effect of $\mathrm{NaCl}$ on proline accumulation in potato seedlings and calli. Acta Physiologiae Plantarum, 26: 263-270.

Rahman, M.H, R. Islam, M. Hossain and S.A. Haider (2008). Different response of potato under sodium chloride stress conditions in vitro. University of Rajshahi, Journal of Bio-Science, 16: 79-83.

Sadasivam, S. and A. Manickam (1991). Amino acids and proteins. In biochemical methods for agricultural sciences (Wiley eastern limited and Tamil Nadu Agricultural University, Coimbtore). pp 33-95. 
Sajid, Z.A. and A. Faheem (2014). Plant regeneration from in vitro-selected salt tolerant callus cultures of (Solanum tuberosum L.). Pakistan Journal of Botany, 46(4): 1507-1514.

Shaterian, J., D.R. Waterer, H. De Jong and K.K. Tanino (2008): Methodologies and traits for evaluating the salt tolerance in diploid potato clones. American Journal of Potato Research, 85: 93-100.

Silva, J.A.B., W.C. Otoni, C.A. Martinez, L.M. Dias and M.A.P. Silva (2001). Microtuberization of Andean potato species (Solanum spp.) as affected by salinity. Scientia Horticulturae, 89: 91-101.

Sudhersan, C., S. Jibi Manuel, J. Ashkanani and A. AlAjeel (2012). In vitro screening of potato cultivars for salinity tolerance. AmericanEurasian Journal of Sustainable Agriculture, 6: 344-348

Velasquez, B., M. Balzarini and E. Taleisnik (2005). Salt tolerance variability amongst Argentine
Andean potatoes (Solanum tuberosum L. subsp. andigena). Potato Research, 48: 59-67.

Yu-Jie, C., Xi-P. Deng, Sang-S. Kwak, W. Chen and A.E. Eneji (2013). Tolerant potato cultivar selection under multiple abiotic stresses. Journal of Food, Agriculture and Environment, 11(2): 760-766.

Zhang, Y. and D. Donnelly (1997): In vitro bioassays for salinity tolerance screening of potato. Potato Research, 40: 285-295.

Zhang, R., G. Shang, M. Meng, F. Men, H. Li and J. Guo (2007). Effects of $\mathrm{NaCl}$ stress on antioxidant defense system of potato. Chinese Potato Journal, 21: 11-14.

Zhang, Z., B. Mao, H. Li, W. Zhou, Y. Takeuchi and K. Yoneyama (2005). Effect of salinity on physiological characteristics, yield and quality of microtubers in vitro in potato. Acta Physiologiae Plantarum, 27: 481-489.

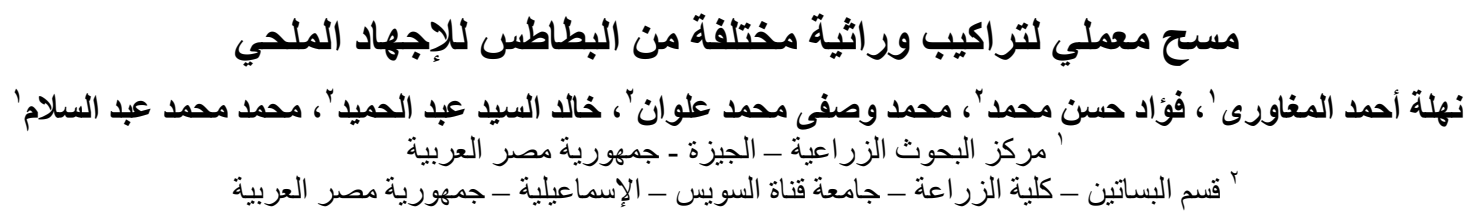

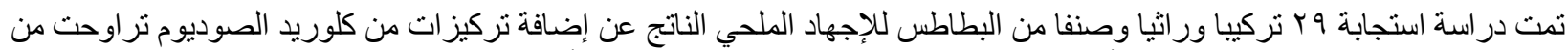

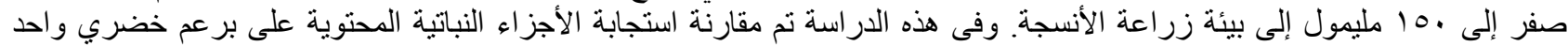

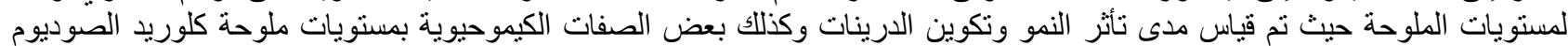

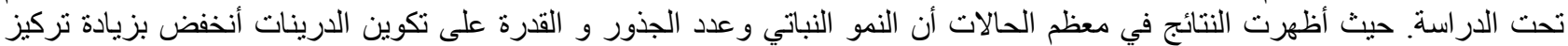

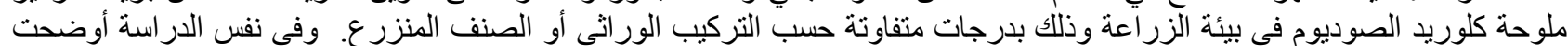

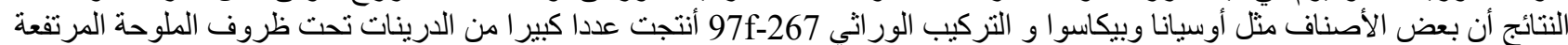

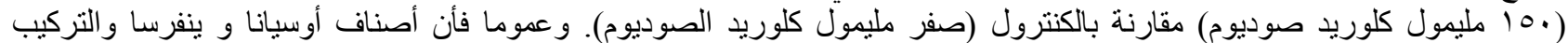

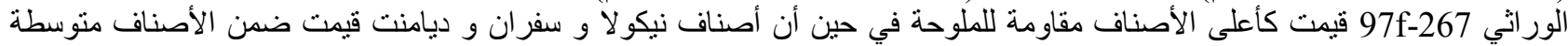

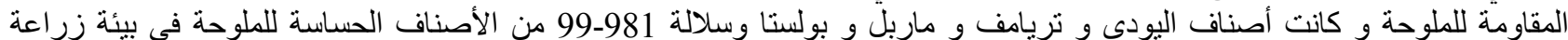

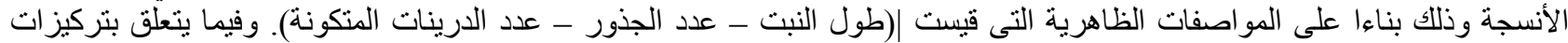

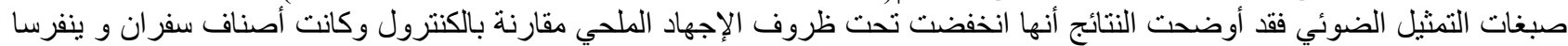

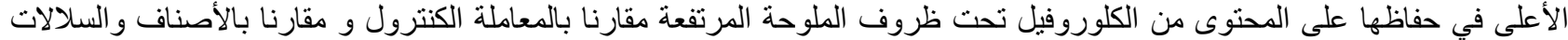

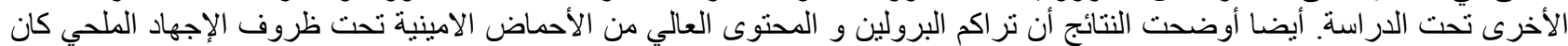

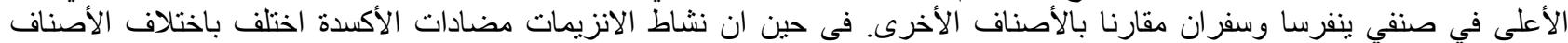

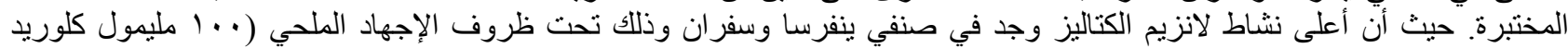

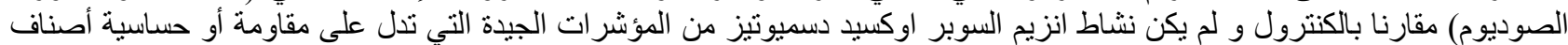

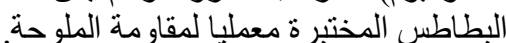

